\title{
THE THEORY OF SOLUBILITY
}

\author{
BY D. TYRER
}

The maximum amount of a substance that a liquid will hold in homogeneous solution at a given temperature, or as it is termed, the solubility of that substance in the given liquid, represents a physical constant which, as yet, it has been found impossible to connect in any consistent manner with any other physical constants or properties of either the solvent or the solute. It is recognized now that this failure to interpret solubility in other terms is due in the case of aqueous solutions, which have received by far the greatest amount of attention, to the complexity of factors coming into play. Before the ideas of hydrates and ions were put forward the dissolution of a salt in water appeared to consist simply of the admixture of the two kinds of molecules, due to an attractive force existing between them, and it was reasonable to expect that the maximum degree of admixture would be found to be related in some way to the properties of the particular solute. Except for a few indefinite generalizations applicable only to closely related substances, the whole accumulated mass of data on the solubilities of substances in water remains an unorganized collection of figures. ${ }^{1}$ The failure to find any relationships is generally supposed to be due to the disturbing effects of ionization and solvate formation. Solutions of normal ${ }^{2}$ organic solutes in normal unassociated organic solvents do not present such difficulties. Such solutions appear to be simply molecular mixtures of the two substances. For example, there is not the slightest amount of evidence to suppose that a solution of naphthalene in benzene is anything else but a mixture of normal molecules of naphthalene and normal molecules of benzene. Yet in such cases as with

\footnotetext{
1 Thermodynamic relations are here excepted as not being concerned with the molecular properties of the substances.

${ }^{2}$ Normal substances are defined as those which consist of simple molecules only. Normal solutions contain no ions, associated molecules or solvates.
} 
aqueous solutions the solubility seems to be a quantity quite unrelated to anything else.

A normal solution can only be regarded as a molecular mixture of the two substances just as a mixture of two gases is merely a molecular mixture. It would be possible in fact to pass continuously from a mixture of two gases to a true solution by merely altering the external conditions, in the following manner. Starting with a mixture of two gases $\mathrm{A}$ and $\mathrm{B}$, we could by compression and cooling, pass continuously through the critical temperature of the mixture, into the liquid state. Then by further cooling, we could reach a point at which the constituent $B$ say, began to separate out in the solid state. We should then have obtained a saturated solution of the solid solute $B$ in the solvent $A$. The possibility of such an experiment makes it clear that we cannot draw up any constitutional difference between a normal solution and a mechanical mixture of molecules. But the mixing of two gases cannot in any true sense be regarded as a process of dissolution, nor can we for this reason legitimately describe air as a solution of oxygen in nitrogen. In a solution the mixing of the constituents is brought about by forces of attraction between the molecules of solvent and solute whereas the mixing of two gases under normal conditions consists simply in the mechanical diffusion of the molecules of the one gas among the molecules of the other. This mechanical diffusion is of course due to the rapid and unrestrained motion of the molecules, and must therefore come into play to some extent in the dissolution of a solute. With easily volatile and slightly miscible components, it is probably a considerable factor. For example, mercury is quite insoluble in bromnaphthalene, yet when heated to a temperature of about $180^{\circ}$ mercury will distil through a supernatant layer of bromnaphthalene just as easily as it does in. vacuum. The mercury vapor mixes with the liquid bromnaphthalene just as it does with a gas. It is not a process of true dissolution. The difference between, say, a mixed gas. and a true solution may be viewed from another aspect. 
Both are molecular mixtures. But in a solution there is a tendency for the molecules of the solute to coalesce and fall out of solution whereas in a mixed gas there is no tendency for either constituent to separate out. But all differences resolve themselves to the fact that in a solution the solute is maintained in the dissolved state by forces of attraction, whereas in a mixed gas under ordinary conditions there is little or no molecular attraction.

In the case of a solid solute and liquid solvent, the tendency of the molecules to diffuse into one another in a manner due solely to the motion of those molecules which have sufficient energy to escape the attraction of the parent substances, is negligibly small. But in the case of a gas and a liquid it is considerable and if the gas is only slightly soluble it is the chief factor involved. Indeed, the validity of Henry's law must depend solely upon this factor. This can be shown in the following manner.

Suppose we have a saturated solution of a slightly soluble gas in contact with excess of gas at constant temperature. Consider unit surface of the liquid. Now if the molecules of gas diffuse freely into and out of the liquid, unrestrained by any forces of attraction, we should have, that the rate of diffusion of gas molecules into the liquid is proportional to the number contained in unit volume of the gas, and the rate of diffusion out again is proportional to the concentration in the liquid. Or, if $\mathrm{N}$ is the number of molecules in unit volume of the gas and $n$ the number of the dissolved gas molecules in unit volume of the liquid

$$
\mathrm{KN}=k_{1} n
$$

where $\mathrm{K}$ and $k_{1}$ are constants. This is Henry's law. If on the other hand the passage of the gas into the liquid were due to molecular attractions alone and were therefore a process of true dissolution, the amount of gas dissolved by a liquid would reach a maximum which would be very little changed by the compression of the undissolved gas. With the more soluble gases both processes occur together and Henry's law is therefore violated. 
It may be concluded then that the formation of a homogeneous solution of a solute in a solvent is due to two factors.

(I) A process of true dissolution due to molecular forces of attraction.

(2) Simple mechanical diffusion of the molecules due to their rapid motion and not associated with energy changes due to molecular attraction.

Except with gases and easily volatile liquids the second factor is negligibly small.

It may be added that the foregoing remarks are equally applicable to solid solutions. The only real difference between a solid solution and a liquid solution is that in the former the molecules, owing to their closeness of packing, must have comparatively little motion and as a consequence the rate of dissolution is extremely slow.

\section{The Factors Determining the Solubility of a Solute in a Solvent.}

The solubility of a solute in a solvent has been found experimentally to depend upon the following factors:

(I) The nature of the solute and solvent.

(2) The temperature.

It is the object of this paper to draw attention to a hitherto unrecognized factor which plays a very considerable part and renders the term "solubility" as at present understood purely arbitrary and destroys any physical meaning which may have been applied to the term.

It was pointed out above that the chief factor causing dissolution was an attractive force between the molecules of solute and solvent. Since the force of attraction acting on a molecule by a surrounding group of molecules is dependent on the distance of separation of the molecules, it is to be expected that the solubility of a solute will be infuenced by the density of the solvent. It was pointed out by the author ${ }^{1}$ that the solubility of a solute depends not only upon the temperature and nature of the solvent but also upon the con-

${ }^{1}$ Jour. Chem. Soc, 97, 1778 (1910). 
centration of the solvent in the solution, that is, upon the mass of solvent contained in unit volume of the solution. It is in a sense an extension of the law of mass action to solubility. This idea was experimentally established ${ }^{1}$ in cases of solutions heated to a temperature above the critical temperature of the saturated solution, when the concentration of the solvent could be varied isothermally. It was observed that the solubility increases with increase of the concentration of the solvent. The recognition of this factor proves that the solubility of a substance in a solvent at a particular temperature, without reference to the density of the solution, is a quantity quite devoid of any theoretical importance and without any definite meaning. It is this indefinite nature of the present conception of solubility that it is the chief object of this paper to emphasize.

The effect of "solvent-concentration" on the solubility of a solute can be best understood by an example. Suppose we take a certain amount of alcohol vapor at $300^{\circ}$ under the ordinary atmospheric pressure and bring it in contact with a little sodium iodide. None of the sodium iodide will be observed to dissolve. But if the alcohol vapor be compressed the sodium iodide will begin to be appreciably soluble and when, for example, the solvent concentration is such that I cc contains 0.3 gram of alcohol the solubility of the sodium iodide will be approximately 4.8 percent. The solubility continues to increase with further compression. Numerous other cases in which the solubility of a solute has been directly observed to increase as the concentration of the solvent increases might be mentioned but the reader is referred to the author's paper quoted above. The insolubility of, say, a salt in steam and its solubility in water at $100^{\circ}$ is a case of the effect of solvent-concentration. If a solvent be diluted with a liquid in which a given solute is insoluble the solubility in the solvent is always diminished. ${ }^{2}$

${ }^{1}$ Tyrer: Jour. Chem. Soc., 97, 621 (1910).

${ }^{2}$ Ibid., 97, 1778 (1910). 
The meaning and importance of solvent-concentration as a factor determining solubility can be best shown graphically.

The construction of the accompanying figure, Fig. I, although it represents a purely imaginary case, is based on the results of the author's measurements of solubilities of salts in solvents in the neighborhood of the critical temperature.

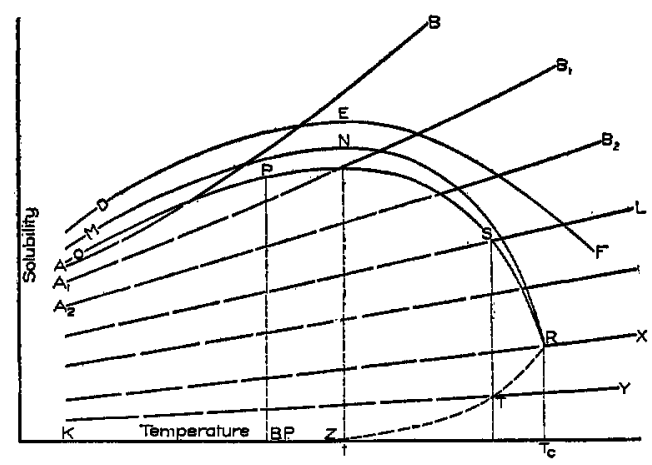

Fig. I

The curves $A B, A_{1} B_{1}, A_{2} B_{2} \ldots \ldots$ etc., represent solubility curves for constant concentrations of the solvent. For example, if we confine the solution in a closed space so that its volume does not change on heating, then the solubilitytemperature curve will be represented by a line similar to the lines $A B, A_{1} B_{1}$, etc. That the solubility of a normal solute in a normal solvent at constant solvent-concentration, increases with the temperature must follow from the fact that the solubility, under ordinary conditions, increases with the temperature although the concentration of the solvent in the solution decreases. The higher lines in the diagram refer to greater solvent-concentrations. The curve OPSR represents the ordinary solubility curve, the solution being heated under constant atmospheric pressure to the boiling point and from the boiling point to the critical temperature under its own vapor pressure.

When a saturated solution is heated in contact with excess of solute, the solvent-concentration decreases. At tem- 
peratures below the boiling point the rate of the decrease is comparatively small but at higher temperatures it becomes greater, and just before the critical temperature of the saturated solution is reached, it becomes very rapid. This causes the solubility near the critical point, to fall rapidly. With solutes which are not appreciably volatile before the critical temperature is reached (salts for example) it may be taken as highly probable that the solubility under a pressure equal to the vapor pressure of the saturated solution, decreases greatly before the critical temperature is reached. With fairly volatile solutes, however, such as organic substances, the solubility will always increase with rise of temperature and will eventually, either before or after the critical temperature of the solution has been reached, become infinitely great. The diagram represents a case of a non-volatile solute.

Suppose we have a sealed glass tube about half filled with a solvent and containing also excess of a non-volatile solute. As the tube and contents are slowly heated more and more of the solute will dissolve, until a point of maximum solubility is reached at, say, the temperature $t$. Further heating will cause some of the dissolved solute to be precipitated. If now the tube contains neither too much nor too little liquid, the solution will pass continuously through the critical temperature $T_{c}$ at which point there is a break in the solubility curve. With further heating the solubility in the one homogeneous phase passes along the curve $\mathrm{RX}$. If the tube contains a relatively small amount of liquid, then before the critical temperature is reached it evaporates completely, say, at the point $S$ on the curve. The solubility, now in the homogeneous vapor phase, will pass along the curve TY. If, on the other hand, the tube contains a relatively large amount of liquid, then at some point on the curve, say, at $\mathrm{S}$, the liquid, owing to its expansion, will just completely fill the tube and the solubility will then pass along the curve SL, increasing with rise of temperature.

As has been already mentioned the ordinary solubility curve as far as the boiling point refers to a constant pressure 
of one atmosphere. If the solution is compressed the solventconcentration increases and the solubility therefore increases. We may have therefore another series of curves which represent the solubility at different temperatures, each at a constant external pressure. For example, suppose a constant pressure is maintained on the solution equal to the critical pressure of the saturated solution, then we should find the solubility represented by a higher curve MNR. Under a constant pressure of, say, Iooo atmospheres we should obtain another curve $\mathrm{DEF}$.

It will be clearly seen that the ordinary solubility curve is quite arbitrary and meaningless. It represents neither the solubility at constant solvent-concentration nor at constant pressure except for the portion below the boiling point.

It may be mentioned that the region OPRZK in the diagram is not practically attainable. The curves in the region of the critical temperature have been experimentally obtained in two cases, though they were not strictly normal solutions, because solvates were probably formed in solution. But still the formation of solvates would not alter the general form of the solubility diagram but would only change the slope of the curves. The diagram is typical and is a complete solubility diagram. The curves for constant solvent-concentrations may be termed solubility isochors and those at constant pressure solubility isobars.

The solubility of any solute in a solvent is completely determined by two variables, temperature and solventconcentration

$$
S=\varphi(T, C)
$$

where $\mathrm{S}$ is solubility, $\mathrm{T}$ is the temperature and $\mathrm{C}$ the concentration of the solvent. $\mathrm{C}$ could, it may be mentioned, be replaced by a function of the density of the solution, but the function here adopted is the more rational. To completely determine this equation it is necessary to find experimentally the relationship between the solvent-concentration and the solubility when the temperature is maintained constant and then to determine the solubility as the temperature varies. 
Suppose we have a particle of a solute surrounded by its saturated solution in a particular solvent. On account of the attractive forces of the surrounding solvent molecules there will be a tendency for the molecules of solute on the surface of the particle to dissociate themselves from the rest of the solute and to pass into solution. But in opposition to this dissolving force and in equilibrium with it, is a molecular force of attraction acting between the molecules of solute cn the surface of the particle and those in the interior of the particle. Although a free interchange of solute molecules must go on between the solution and the undissolved solute we can quite legitimately imagine there to be a state of static equilibrium, the molecules on the surface of the particle being just prevented from dissolving by the inner attractive force of the other molecules of the solute. For a particle whose diameter is greater than the diameter of the sphere of attractive influence of a molecule, the latter force will be a function of the density of the solute. If we suppose molecular attraction to be independent of the temperature and if the force between two molecules, the distance between the centers of which is $\mathrm{S}$, can be represented by

$$
\frac{\mathrm{K}}{\mathrm{S}^{n}}
$$

where $\mathrm{K}^{1}$ is a constant ${ }^{1}$ dependent on the nature of the sub-

- stance and $n$ is a small numeral constant for all substances, then we can represent the inner force of attraction acting on a molecule at the surface of an undissolved particle of solute by

$$
\frac{\mathrm{C}}{v^{n_{1}}}
$$

where $\mathrm{C}$ is a constant, and $v$ is the specific volume of the solute. The dissolving force or the force which causes the molecules of solute to pass into solution is made up of two

${ }^{1}$ It would be too long to give a proof here of this term, the reader may be referred for a treatment of molecular attraction to the papers by J. E. Mills: Jour. Phys. Chem., 6, 209 (I902); 8, 383 (I904); 9, 402 (I905); I0, I (Ig06). 
parts: (I) The attraction of the molecules of solvent on the molecules of the solute; (2) the attraction of the molecules of solute already dissolved. The first of these quantities will depend upon the total volume occupied by the solution containing a constant amount of the solvent, and it may be expressed as

$$
\frac{\mathrm{C}_{1}}{\mathrm{~V}^{n_{1}}}
$$

$\mathrm{V}$ being the total volume of the solution. The second quantity will depend upon the concentration of the molecules of the solute in the solution and may be written

$$
\mathrm{C}_{2}\left(\frac{\mathrm{N}}{\mathrm{V}}\right)^{n_{1}}
$$

where $\mathrm{N}$ is the number of molecules dissolved.

We must have then according to our principle the equation

$$
\frac{\mathrm{C}}{v^{n_{1}}}=\frac{\mathrm{C}_{1}}{\mathrm{~V}^{n_{1}}}+\frac{\mathrm{C}_{2}}{\mathrm{~V}^{n_{1}}}\left(\frac{\mathrm{S}}{\mathrm{M}}\right)^{n_{1}}
$$

where $S$ is the solubility and $M$ the molecular weight of the solute.

This gives

$$
a\left(\frac{\mathrm{V}}{v}\right)^{n_{1}}-b=\mathrm{S}^{n_{1}}
$$

where for a given solute and solvent $a, b$, and $n$ are constants. But $V$ is a function of the specific volume of the solvent and of the solubility. Now it has been shown experimentally that the volume of a solute in solution, is, in normal cases, approximately equal to the volume occupied by the pure liquefied solute at the same temperature. ${ }^{1}$

We may write then

$$
\mathrm{V}=\mathrm{W} v_{l}+\mathrm{S} v_{s}
$$

where $v_{l}$ is the specific volume of the pure solvent, $v_{s}$ the specific volume of the solute in solution and $S$ the amount of solute in $W$ parts of the solvent. $S$ is then, the solubility when we put $W=$ roo.

\footnotetext{
${ }^{1}$ See Tyrer: Jour. Chem. Soc., 97, 2620 (1910); 99, 87 I (191 I).
} 
Our equation of equilibrium then becomes

$$
a\left(\frac{\mathrm{I} O 0 v+\mathrm{S} v_{s}}{v}\right)^{n_{1}}-\mathrm{S}^{n_{1}}=b
$$

In this equation $a$ and $b$ are constants, $s$ the solubility, $v_{l}$ and $v$ the specific volumes of the pure solvent and pure solute respectively and $v_{s}$ the specific solution volume of the solute.

It cannot be tested quantitatively, as we do not know $n_{1}$ and in any case experimental data are much too incomplete. It may, however, be applied qualitatively.

Take as the first case a gaseous solute and liquid solvent. If the solubility is small we can neglect the term $\mathrm{S} v_{s}$.

With rise of temperature $v$ increases at a greater rate than $v_{l}$ and hence the solubility must diminish. Increase of pressure for similar reasons should cause the solubility to increase. With a liquid solute the solubility may either increase or decrease with the temperature. As a matter of fact practically all really normal liquids are miscible in all proportions. With a solid solute since $v_{l}$ increases at a greater rate than $v$, rise of temperature should always cause (in normal cases of course) the solubility to increase. Until experimental data are available nothing more can be done to test the validity of the above equation.

A few remarks may be made here regarding infinite solubility. With a solid solute and a liquid solvent it is obvious on account of their physical difference that a solid can never become infinitely soluble at a temperature below its ordinary melting point. When two normal liquids are mixed very little heating effect is produced. This appears to be quite a general rule. Moreover very little change in volume occurs. This clearly means that little or no work is done in the dissolution of a normal liquid solute in a normal liquid solvent, and from this we should expect that normal liquids are miscible in all proportions. And I do not know of a single case in which two normal liquids are only partially miscible. If this rule is quite general then it follows, that with normal solid solutes, the solubility in a normal solvent 
increases with the temperature and becomes infinitely soluble at its melting point. Sufficient experimental data exist in a few cases to test the accuracy of this suggestion. In the following table are given the temperatures at which the specified solutes become infinitely soluble. These temperatures have been found by extrapolating the solubility curves, the solubility being expressed in parts per Ioo of saturated solution. The solubility data were taken from Seidell's dictionary of solubilities. In the last column are given the melting points of the solutes.

\begin{tabular}{|c|c|c|c|}
\hline Solute & Solvent & $\begin{array}{l}\text { Temperature } \\
\text { of infinite } \\
\text { solubility }\end{array}$ & $\begin{array}{l}\text { Melting } \\
\text { point of } \\
\text { solute }\end{array}$ \\
\hline Naphthalene & $\begin{array}{l}\text { Carbon tetrachloride } \\
\text { Carbon bisulphide } \\
\text { Benzene } \\
\text { Chlorobenzene } \\
\text { Hexane }\end{array}$ & $\begin{array}{l}80^{\circ} \\
78^{\circ} \cdot 5 \\
79^{\circ} \cdot 5 \\
79^{\circ} \cdot 5 \\
79^{\circ}\end{array}$ & $80^{\circ}$ \\
\hline Triphenylmethane & $\begin{array}{l}\text { Benzene } \\
\text { Carbon bisulphide } \\
\text { Hexane } \\
\text { Chloroform } \\
\text { Pyridine } \\
\text { Pyrrol } \\
\text { Thiophen }\end{array}$ & $\begin{array}{l}94^{\circ} \\
94^{\circ} \\
92^{\circ} \\
95^{\circ} \\
92^{\circ} \cdot 5 \\
93^{\circ} \cdot 5 \\
93^{\circ} \cdot 5\end{array}$ & $92^{\circ}$ \\
\hline$m$-Dinitrobenzene & $\begin{array}{l}\text { Benzene } \\
\text { Chloroform }\end{array}$ & $\begin{array}{l}92^{\circ} \\
88^{\circ}\end{array}$ & $89^{\circ}$ \\
\hline$p$-Dibrombenzene & $\begin{array}{l}\text { Ether } \\
\text { Carbon bisulphide } \\
\text { Benzene } \\
\text { Brombenzene }\end{array}$ & $\begin{array}{l}84^{\circ} \\
84^{\circ} \\
84^{\circ} \cdot 5 \\
88^{\circ}\end{array}$ & $89^{\circ}$ \\
\hline Anthraquinone & Ether & $\begin{array}{l}275^{\circ} \\
\text { (experimental) }\end{array}$ & $285^{\circ}$ \\
\hline
\end{tabular}

Considering the approximate character of the figures there is a fairly close agreement between the temperatures of infinite solubility and the melting points. In the case of dibrombenzene the temperatures of infinite solubility is somewhat lower than the melting point. This can only be due to errors for although a solute does not necessarily become 
infinitely soluble at the melting point it cannot clearly attain this point below the melting point.

Given two normal liquids, some condition relative to their properties must be determinable upon which depends the possibility of their mixing in all proportions. It can be shown on the basis of molecular attraction that when a liquid solute is added gradually to a liquid solvent, both being normal, that the total force, in virtue of which the solute tends to dissolve, first decreases, reaches a minimum and then increases. If the total force acting against the dissolution tendency be less than this minimum value, the two liquids will be miscible in all proportions.

It has already been shown that the dissolution of a particle of a solute in a liquid is due to ( $I$ ) the attraction exercised by the molecules of the solvent (2) the attraction exercised by the molecules of solute already in solution. The total force of attraction inducing dissolution may be written

$$
\frac{\mathrm{C}_{1}}{\mathrm{~V}^{n_{1}}}+\frac{\mathrm{C}_{2} \mathrm{~S}^{n_{1}}}{\mathrm{~V}^{n_{1}}}
$$

where $C_{1}$ and $C_{2}$ are constants, $v$ is the total volume of the solution containing say I gram of solvent and $S$ is the mass of solute in the solution. The force of attraction acting between the molecules of the undissolved solute and restraining the dissolution may be expressed

$$
\frac{\mathrm{C}}{v^{n_{1}}}
$$

where $\mathrm{C}$ is a constant and $v$ is the specific volume of the solute. If the solution is unsaturated then

$$
\frac{\mathrm{C}_{1}}{\mathrm{~V}^{n_{1}}}+\frac{\mathrm{C}_{2} \mathrm{~S}^{n}}{\mathrm{~V}^{n_{1}}}>\frac{\mathrm{C}}{v^{n_{1}}}
$$

The problem is to determine how the expression on the left varies as the solubility increases.

Now since in a normal solution of two liquids no change in volume occurs when they are mixed we may write

$$
\mathrm{V}=v_{t}+\mathrm{S} v_{s}
$$


where $v_{l}$ is the volume of the I gram of the solvent and $v_{s}$ the specific volume of the solute.

Therefore, the above expression becomes

$$
\frac{\mathrm{C}_{1}}{\left(v_{l}+\mathrm{S} v_{s}\right)^{n_{1}}}+\frac{\mathrm{C}_{2} \mathrm{~S}^{n}}{\left(v_{l}+\mathrm{S} v_{s}\right)^{n_{1}}}>\frac{\mathrm{C}}{v_{s}^{n_{1}}}
$$

It can readily be shown by differentiation that the expression on the left is a minimum when

$$
\mathrm{S}=\left(\frac{\mathrm{C}_{1}}{\mathrm{C}_{2}} \cdot \frac{v_{s}}{v_{l}}\right) \frac{1}{n_{1}-1}
$$

Therefore, the required condition for complete miscibility or infinite solubility is that

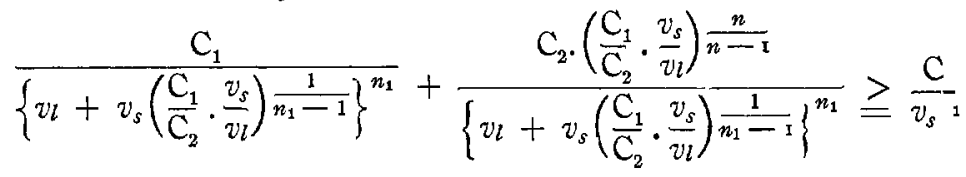

It would be impossible to deal quantitatively with this equation without a knowledge of the law of molecular attraction. But it suffices to show the possibility of treating the phenomenon of solubility from a mathematical basis.

\section{On the Relative Solvent Powers of Different Liquids for a Given Solute}

The solubility of a solute in a solvent depends of course on the nature of both substances, but by making the solute constant we cause it to depend solely upon the solvent. We thus obtain a conception of relative solvent power. The solvent power of a liquid is measurable by the solubility of the particular solute. But the solubility depends upon the two conditions; temperature and solvent-concentration. We may define then the solvent power of a liquid towards a particular solute at a given temperature, as the solubility of the solute in the solvent at unit solvent-concentration. And the molecular solvent power may be defined as the solubility when unit volume contains I gram molecule of the solvent.

As temperature rises the solvent power of a liquid for a particular solute (normal cases only are considered) in- 
creases. Eventually the solute becomes infinitely soluble, when the solvent power may be regarded as becoming infinitely great.

The liquid which will dissolve most of the given solute has not necessarily the greatest solvent power, for the solvent concentration must also be taken into account in making comparisons.

For comparatively small differences in the concentration of a solvent we may regard the solubility as a linear function of the solvent-concentration and we can thus obtain an approximate idea of the relative solvent powers of various normal liquids for a given solute. In the following table are given the solvent powers of four liquids for naphthalene at $20^{\circ}$ calculated in this way:

\begin{tabular}{l|c|c|c}
\hline Solvent & $\begin{array}{c}\text { Grams of naphtha- } \\
\text { lene dissolved by } \\
\text { I gram molecule } \\
\text { of solvent }\end{array}$ & $\begin{array}{c}\text { Molecular concentra- } \\
\text { tion of solvent in } \\
\text { saturated solution }\end{array}$ & $\begin{array}{c}\text { Molecular solvent } \\
\text { power at a con- } \\
\text { stant molecular } \\
\text { solvent concen- } \\
\text { tration of } 0.00854\end{array}$ \\
\hline Chloroform & 37.9 & 0.00854 & 37.9 \\
Toluene & 25.7 & 0.00724 & 30.4 \\
Benzene & 43.9 & 0.00762 & 49.2 \\
Hexane & I4 & 0.00712 & 16.8
\end{tabular}

Benzene would appear to be the best solvent (in the present sense of the word) for naphthalene and hexane the worst of the four.

It would be very interesting to determine in this manner the relative solvent powers of the above and other liquids for other solutes and to see whether or not they fall into the same order, and it would also be interesting to determine whether the order of arrangement was affected by temperature, but unfortunately insufficient experimental data are available.

The advancement and general recognition of the hydrate theory and of the theories of dissociation and molecular association have rendered the further study of solubilities in associated solvents like water and the alcohols, quite useless 
from the point of view of the theory of solubility. In this sense it is unfortunate that so much work should have been done on aqueous solutions in which a hopeless complication of factors comes into play, and so little done with unassociated organic substances which present cases far simpler in character.

There is one question, however, which must precede a quantitative study of solubility and that is the determination of the law governing molecular attraction, and until this problem is solved there can be little hope of completely understanding the process of dissolution. And this is self-evident when one considers that the dissolution of a solute is caused by molecular attraction, and that the solute is held in solution by the same forces. Solubility is, in fact, but a problem of molecular attraction.

\section{Addendum}

Since writing the above the author's attention has been brought to a paper by Hardman and Partington ${ }^{1}$ in which some views of the author on the subject matter of the present paper, are criticised. Objection is raised to the conception of the solvent-concentration as a factor influencing solubility. They consider the simple equation

$$
\mathrm{S}=\phi(T)
$$

where $S$ is the solubility and $T$ the temperature, to be sufficient and to include all possible factors. But it cannot clearly be true because when the temperature is constant the solubility may still vary with the density of solution. This variation is moreover experimental and quite free from any hypothetical assumptions. It might also be urged that the variation of solubility when the solvent density is changed could be interpreted in terms of the external pressure. But this would be unsatisfactory because a liquid and saturated vapour both are under the same external pressure yet do not dissolve the same amounts of a common solute. We could however, write the solubility at constant temperature, as a function of the

\footnotetext{
${ }^{1}$ Jour. Chem. Soc., 99, I769 (I9II).
} 
density of the saturated vapour but it is more rational to refer it to the concentration of the solvent, since it is chiefly a property of the solvent.

A few remarks may be made here in regard to equations representing the variation of solubility and variation of the temperature. It is obvious from the foregoing that no such equation is complete which does not take account either of the density of the solution or the concentration of the solvent in the solution. R. Hardman and Partington have reduced the following equation

$$
\log \mathrm{S}=\mathrm{A}-\frac{\mathrm{B}}{\mathrm{T}}-\mathrm{C} \log \mathrm{T}
$$

where $\mathrm{S}$ is the solubility $\mathrm{A}, \mathrm{B}$ and $\mathrm{C}$ are constants. Several of the asumptions however on which this equation is based, are quite erroneous. For example it was assumed that the specific heat of a liquid is constant over a moderate range of temperature. In the case of benzene which was the solvent used in the cases employed to test the equation, the specific heat varies about i 2 percent over a range of about 70 degrees. The equation however holds fairly closely. But thus it must clearly do on account of its general character. It contains three constants and is similar in character to the equation

$$
\mathrm{S}=\mathrm{A}+\mathrm{BT}+\mathrm{CT}^{2}
$$

which holds equally well. The most elementary study of solubility curves, will show the impossibility of representing the relation between solubility and temperature by a general equation of a simple character. There is probably no solubility equation containing less than three constants all of which depend on the nature of the solution and an equation with three unknown constants is of little practical value.

The Chemistry' Department, The University, Manchester 